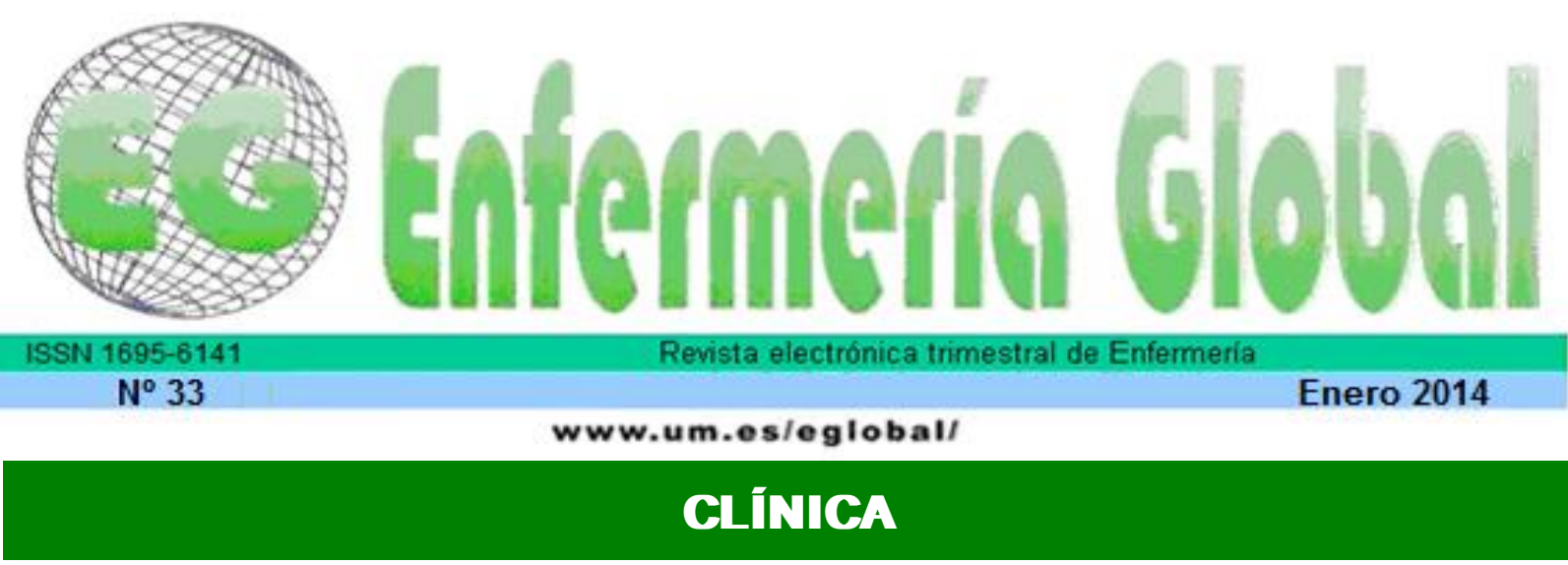

\title{
Madres adolescentes, un reto frente a los factores que influyen en la lactancia materna exclusiva
}

Adolescent mothers a challenge facing the factors influencing exclusive breastfeeding

\begin{abstract}
*Pinilla Gómez, Elvinia ${ }^{* *}$ Domínguez Nariño, Claudia Consuelo **García Rueda, Alexandra

*Profesora Titular Escuela de Enfermería. Directora Grupo de Investigación GRINFER. E-mail: elvinia2000@yahoo.com **Profesora Titular Escuela de Enfermería. Grupo de Investigación GRINFER. Universidad Industrial de Santander. Colombia.
\end{abstract}

Palabras clave: lactancia materna; factores; madres, adolescentes.

Keywords: breast feeding; factors; mothers; adolescents.

\section{RESUMEN}

Objetivo: Determinar los factores que influyen en la práctica de la lactancia materna exclusiva en madres adolescentes.

Material y Método: Estudio de corte transversal, con 158 madres adolescentes en etapa de lactancia que asisten a los servicios de primer nivel de atención de Bucaramanga. Se aplicaron dos instrumentos para caracterizar las madres adolescentes y el APGAR familiar para evaluar la funcionalidad familiar. Datos digitados y validados en Epi- Info 6.04d y analizados en Stata 10.1; para analizar las variables continuas, se utilizaron medidas de tendencia central, las variables categóricas fueron expresadas como proporciones. El análisis multivariado se describió mediante razón de prevalencia e intervalo de confianza del $95 \%$.

Resultados: Promedio de edad 17.9 años, el 39.4\% lactó 6 meses y el 9.8\% lactó más de 6 meses; el $89.2 \%$ ya habían tenido hijos. Las razones por las cuales no alimenta a su hijo con solo leche materna son: el $9.5 \%$ producen poca leche, el $12 \%$ el niño se queda con hambre, y el $20.7 \%$ otras causas. Durante la lactancia el $82.3 \%$ acaricia a su bebé. Las personas que recomiendan la lactancia materna son el $84.3 \%$ médico y enfermera, el $63.3 \%$ un familiar.

Conclusiones: Se precisa del asesoramiento y apoyo familiar y de las instituciones de salud para que el amamantamiento sea una experiencia satisfactoria tanto para la madre adolescente como para el hijo. 


\title{
ABSTRACT
}

Objective: To determine the factors that influences the practice of exclusive breastfeeding in adolescent mothers.

\begin{abstract}
Methods: Cross-sectional study with 158 mothers lactating adolescents attending the services of primary care of Bucaramanga- Colombia. The instruments used to characterize adolescent mothers and to evaluate the effectiveness of family functionality the family APGAR. Data entered and validated in Epilnfo 6.04d and analyzed in Stata 10.1, to analyze continuous variables, we used measures of central tendency; categorical variables were expressed as proportions. Multivariate analysis described by prevalence ratio and confidence interval of $95 \%$.
\end{abstract}

Results: mean age 17.9 years, $39.4 \%$ six months nursed and nursed $9.8 \%$ over 6 months and $89.2 \%$ had already had children. The reasons for not feeding her child with only breast milk are: $9.5 \%$ produce little milk, $12 \%$ child is still hungry, and $20.7 \%$ other causes. During lactation the $82.3 \%$ caresses her baby. People recommend breastfeeding are the doctor and nurse $84.3 \%$, a relative $63.3 \%$ relative.

Conclusions: Accurate counseling and family support from the health institutions that will assure breastfeeding as a satisfying experience for both the teenager mother and the child.

\section{INTRODUCCIÓN}

Actualmente se sigue reconociendo la importancia de la lactancia materna en todo el mundo, especialmente en las comunidades que disponen de menos recursos, donde constituye la única forma asequible de alimentar a sus lactantes.

En las últimas décadas, la lactancia materna se ha transformado en un importante indicador que refleja el nivel de bienestar de una sociedad en su conjunto. En este sentido, la recomendación actual de la OMS para las madres es practicar la lactancia exclusiva desde el nacimiento hasta los seis meses, sin otros líquidos ni alimentos sólidos adicionales que no sean medicamentos o vitaminas. Después de esa edad, es posible introducir alimentos complementarios inocuos y adecuados, pero la lactancia a libre demanda debe continuar hasta que el niño tenga dos años de edad o más ${ }^{(1,2)}$.

La lactancia materna exclusiva en Colombia bajó de 2.2 meses en el 2005 a 1.8 meses en el 2010, presentando serias diferencias en las diferentes regiones del país. Es decir 4\% de los niños menores de 5 años nunca fue amamantado ${ }^{(3)}$. La Encuesta Nacional de 2010 sobre la Situación Nutricional en Colombia (ENSIN), evidencia los avances del país en esta práctica. La encuesta mostró, entre otros aspectos, que en Colombia, el promedio de la lactancia total está en 14.9 meses y de lactancia materna exclusiva es de 1.8 meses y leche materna complementada con otros líquidos nolácteos, fue de 2.7 meses en promedio ${ }^{(4,5)}$.

Los estudios muestran que la mayoría de los niños alimentados con lactancia exclusiva durante los primeros 6 meses de vida alcanzan un óptimo crecimiento y desarrollo, lo que demuestra la importancia de continuar con lactancia materna exclusiva y no sustituirla con leche artificial ${ }^{(1,2)}$.

La evidencia ha demostrado que los componentes de la leche materna tienen la calidad y concentración adecuada para cada niño en particular, que el contacto del niño con su madre durante los primeros meses de vida es indispensable para un buen desarrollo biopsicosocial del individuo y que la función de succionar es el mejor estímulo para un desarrollo morfofuncional armónico de las estructuras máxilo faciales y orofaríngeas. 
El amamantamiento es una experiencia satisfactoria tanto para la madre como para el hijo por el contacto piel con piel que se produce entre ambos, la madre es extraordinariamente sensible durante la lactancia y su mayor preocupación gira en torno a la alimentación y cuidado de su hijo. Sin embargo, en las madres adolescentes, debido fundamentalmente a la etapa de la vida que están viviendo, al desconocimiento y falta de experiencia, les provoca "angustia".

En Colombia el $19 \%$ de la población adolescente son madres ${ }^{(4)}$. La madre adolescente es una mujer joven, necesita vivir esa etapa de su vida, ya que la maternidad temprana no la hace mujer adulta; no está preparada, en un corto tiempo debe lograr importantes metas, asumir su sexualidad, su salud reproductiva, autocuidado, relaciones afectivas con la familia, con su pareja, con su hijo. Son tareas y desafíos que las jóvenes nunca han abordado y llevan a estas madres adolescentes a sentirse recargadas de trabajo y con una carga emocional muy fuerte, lo que constituye un gran riesgo para la Lactancia Materna.

No podemos de dejar de mencionar el "apego", relación madre-hijo, vínculo al que se la ha dado mucha importancia últimamente a nivel nacional y mundial, debido a que un buen "apego" se relaciona con una mayor duración y mejor calidad de la lactancia natural, favoreciendo los lazos afectivos en la relación madre-hijo inicial, lo que estimula un mejor desarrollo psicomotor del niño ${ }^{(6)}$.

En un estudio realizado sobre factores psicosociales que influyen en el abandono de la lactancia materna se encontró que en el abandono de la lactancia materna por grupos de edades, el hecho fue más frecuente en las menores de $20(71.4 \%)$ y no abandonaron el $28.6 \%$ de estas menores ${ }^{(7)}$. En otro realizado en Argentina, se encontró que el abandono de amamantamiento exclusivo constatado en el $42 \%$ de madres incluyó las siguientes causas: trabajo materno 42\%, poca producción de leche $35 \%$, rechazo del bebé al pecho $12 \%$, patología del lactante $7 \%$ y mala preparación de los pezones $4 \%{ }^{(2)}$.

Es esencial conocer las razones que han influenciado a las madres adolescentes para brindar o no alimentación al seno materno, con el fin de dar pautas u orientar el trabajo preventivo de la atención primaria en salud, a favor de la salud materno infantil de la población de estudio.

Por tal razón, el objetivo del presente estudio fue Determinar los factores que influyen en la práctica de la lactancia materna exclusiva en madres adolescentes.

\section{MÉTODO}

Se realizó un estudio de corte transversal, la población de estudio fueron madres adolescentes en etapa de lactancia que asisten a los servicios que ofrecen las unidades operativas de la Empresa Social del Estado Instituto de Salud de Bucaramanga ESE ISABU, de primer nivel de atención. Se calculó un tamaño de muestra en el programa estadístico Epi info versión 6.04d (8) y se analizó en Stata10.1 ${ }^{(9)}$, con los siguientes criterios: nivel de confiabilidad del 95\%, poder del $80 \%$, una prevalencia esperada de no lactancia materna exclusiva en madres adolescentes en el grupo no expuesto del $40 \%$ para poder detectar asociación mayor a una razón de prevalencia $\geq 1.4$ dando como resultado una muestra de 158 madres adolescentes en etapa de lactancia atendidas durante el segundo semestre de 2011, y que residían en el área metropolitana de Bucaramanga. Muestreo por conveniencia 
para la recolección de la información, de donde se tomaron las madres adolescentes que iban a participar, hasta completar la muestra.

Criterios de inclusión: adolescentes gestantes de 13 a 19 años atendidas en la ESE ISABU, el informante es madre adolescente sin alteraciones mentales diagnosticadas por un especialista. Criterios de exclusión: madres adolescentes menores de 13 años, adolescente informante con alteraciones mentales. Se aplicaron dos Instrumentos para la recolección de la información, los cuales cumplen con las propiedades psicométricas que permiten utilizarlos y garantizar la validez del estudio, el primero es para caracterizar las madres adolescentes y el segundo el APGAR familiar para evaluar la funcionalidad familiar ${ }^{(10)}$.

Para la digitación y análisis de datos se hizo una base de datos doblemente digitada en Epi info 6.04d. Luego de revisada la base de datos, fue exportada a Stata10.1, para el análisis de la información. Se realizó el análisis descriptivo de las características de la población. Las variables en escala nominal fueron descritas como porcentajes junto con sus intervalos de confianza del $95 \%$, y las variables continuas se describieron con medidas de tendencia central, posteriormente se hicieron cálculos de medidas estadísticas como: porcentajes, frecuencias, representados a través de tablas. El análisis multivariado se describió mediante razón de prevalencia e intervalo de confianza del 95\%. Los procedimientos del estudio se iniciaron con la identificación de la adolescente encuestada, se realizó el contacto en los centros de salud y hogares de las madres adolescentes. La recolección se hizo durante el segundo semestre del 2011 y primer semestre del 2012.

Es una investigación sin riesgo para los informantes, según la clasificación de la resolución 8430 de 1993 porque no se realizó ninguna intervención o modificación intencionada de las variables biológicas, fisiológicas, psicológicas o sociales de las personas que participan en el estudio. Se tuvo en cuenta la autorización del representante legal de ESE ISABU, Informado de las participantes por escrito, principios de autonomía, privacidad y confidencialidad de la información ${ }^{(11)}$.

\section{RESULTADOS}

Se analizaron 158 encuestas aplicadas a madres adolescentes del área metropolitana de Bucaramanga que se encontraban en periodo de lactancia materna. Las características sociodemográficas y económicas de las encuestadas muestran que la edad promedio de las madres adolescentes fue de 17.9 años, con nivel de escolaridad secundaria en el $82.8 \%$ de las encuestadas, y cuya ocupación fue el hogar para el $62 \%$. El estado civil predominante en un $65.8 \%$ es la unión libre y con respecto a la seguridad social el $70.9 \%$ tienen una EPSS (ARS) que corresponde a Entidades Promotoras de Salud Régimen Subsidiado. El 72.8\% vivían en arriendo y pertenecían a los estratos 1 y 2 con el 56.3\% y $32.9 \%$ respectivamente. El promedio de ingreso económico mensual está dado por un $64.3 \%$ con un salario mínimo (SMLV), mientras que el $24 \%$ de las familias tienen ingresos inferiores a un SMLV. La responsabilidad como cabeza de familia recae en el esposo/compañero en un $46.8 \%$. En el $26.6 \%$ de las familias existe en algún miembro de la familia exceso de trabajo, el cual se distribuye un $41 \%$ en el esposo/compañero, un $15.4 \%$ en la madre de las adolescentes y el $12.8 \%$ en la madre adolescente. 
Tabla I. Variables de descripción de las madres adolescentes (Variables nominales)

\begin{tabular}{lcc}
\hline \multicolumn{1}{c}{ VARIABLES } & $\mathrm{N}^{\circ}$ & $\%$ \\
ESTADO CIVL & & \\
Unión libre & 104 & 65.8 \\
Soltera & 37 & 23.4 \\
OCUPACION & & \\
Hogar & 98 & 62.0 \\
Trabaja & 46 & 29.1 \\
Estudia & 22 & 13.9 \\
ESCOLARIDAD & & \\
Primaria & 19 & 12.1 \\
Secundaria & 130 & 82.8 \\
Tecnología & 4 & 2.55 \\
Universitaria & 4 & 2.55 \\
ESTRATO SOCOECONÓMICO & & \\
Estrato 1 & 89 & 56.3 \\
Estrato 2 & 52 & 32.9 \\
Estrato 3 & 15 & 9.5 \\
Estrato 4 & 2 & 1.3 \\
SEGURIDAD SOCIAL & & \\
EPS & 32 & 20.3 \\
EPSS (ARS) & 112 & 70.9 \\
SISBEN & 10 & 6.3 \\
Ninguno & 4 & 2.5 \\
TIPO DE VVIENDA & & \\
Propia & ${ }^{*}{ }^{\circ}{ }^{\circ}=$ madres adolescentes & 27.2 \\
En arriendo & 115 & 72.8 \\
&
\end{tabular}

Dentro de los antecedentes obstétricos cabe resaltar que el $73.5 \%$ asistieron a controles prenatales oscilando entre 5 y 8 controles durante el periodo de gestación. El $84.2 \%$ de las madres adolescentes tienen un hijo y la vía del parto que predomina es el vaginal con un $54.4 \%$, de los cuales el $89.2 \%$ tuvieron sus hijos a término. Con respecto al número de hijos el $84.2 \%$ tenían 1 hijo, el $14.6 \%$ tenían 2 hijos y el $1.3 \%$ tenían 3 hijos. Ver Tabla II. 
Tabla II. Antecedentes obstétricos de las madres adolescentes.

\begin{tabular}{lcc}
\hline \multicolumn{1}{c}{ VARIABLE } & $\mathrm{N}^{\circ}$ & $\%$ \\
CONTROLES PRENATALES & & \\
1 a 4 & 18 & 11.9 \\
5 a 8 & 111 & 73.5 \\
9 o más & 22 & 14.6 \\
VİA DEL PARTO & & \\
Vaginal & 86 & 54.4 \\
Cesárea & 72 & 45.6 \\
GESTACIONES & & \\
A término & 141 & 89.2 \\
Pretérmino & 17 & 10.8 \\
NÚMERO DE HIJOS & & \\
1 & 133 & 84.1 \\
2 & 23 & 14.6 \\
3 & 2 & 1.3 \\
\hline
\end{tabular}

Los resultados mostraron que dentro de los factores que influyen en el abandono de la lactancia materna, el $39.2 \%$ ha lactado a su hijo con solo leche materna durante 6 meses, el $55.4 \%$ ha incluido agua o alimentos antes de los 6 meses de vida, y las razones por las cuales no alimenta a su hijo con solo leche materna el $9.5 \%$ considera que producen poca leche, el $12 \%$ porque el niño se queda con hambre, y el $20.7 \%$ otras causas (agua, preocupaciones, influencia de otras personas, problemas clínicos).

En relación al tiempo destinado para la lactancia, el 94.3\% tienen el tiempo suficiente para lactar y el $84.3 \%$ lo pueden hacer porque están en el hogar. Las madres adolescentes durante el proceso de la lactancia el $82.3 \%$ acaricia a su bebé, el $80.4 \%$ le habla, el $74 \%$ lo mira y el $15.2 \%$ realiza otras actividades de las cuales el $31.8 \%$ ve televisión, y el $27.3 \%$ juegan mientras lactan. Con respecto a las personas que recomiendan la lactancia materna exclusiva durante los primeros 6 meses de vida del niño, el $84.3 \%$ lo hacen el médico y la enfermera, el $63.3 \%$ un familiar, el $22.8 \%$ una amiga y el $19 \%$ una vecina.

Tabla III. Factores que influyen en la Lactancia Materna Exclusiva.

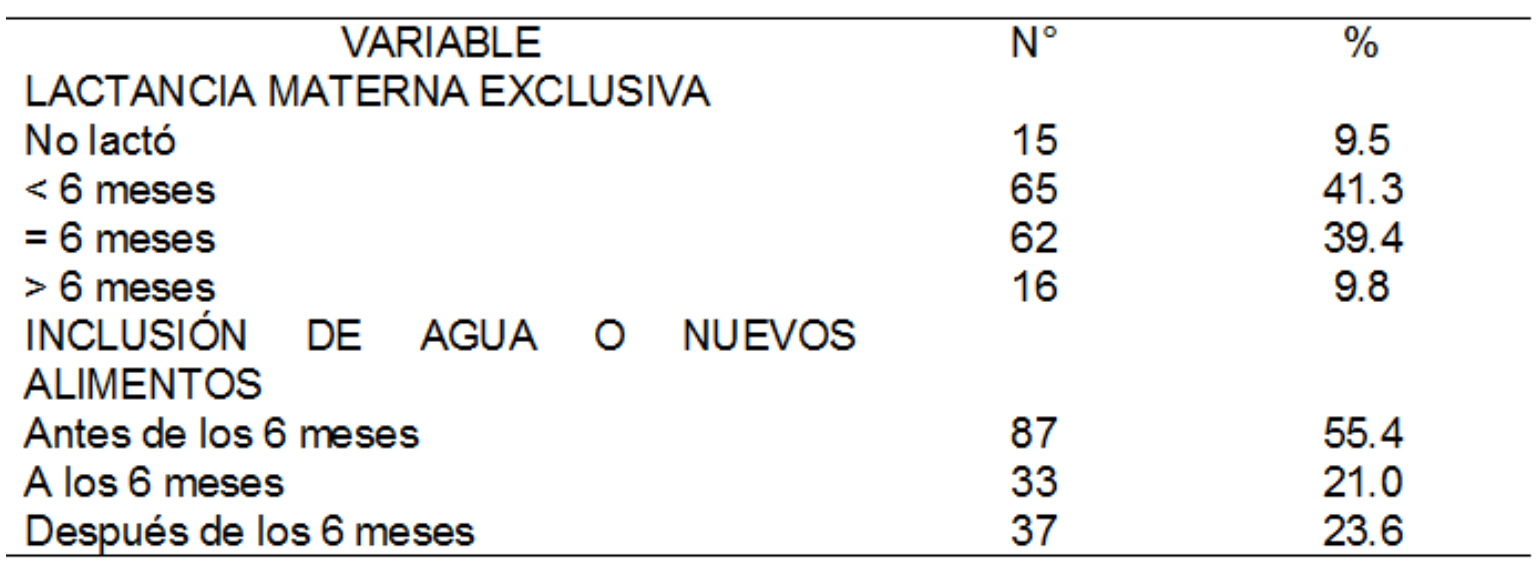

Del análisis multivariado de los factores que influyen en la lactancia materna exclusiva, es importante resaltar tanto el apoyo familiar como el de las instituciones de 
salud, y que la influencia de la familia, que para este estudio estuvo representado por la suegra y por la mamá de la adolescente, se constituyen en factores protectores para la lactancia materna exclusiva, al igual que los sentimientos de alegría al momento de lactar.

Tabla IV. Factores que influyen en la Lactancia Materna Exclusiva (LME). (Modelo multivariado)

\begin{tabular}{lcc}
\hline VARIABLE & $\begin{array}{c}\text { Razón de } \\
\text { Prevalencia }\end{array}$ & IC 95\% \\
Apoyo familiar y centro de salud & 2.2 & $1.0-4.8$ \\
Influencia de la suegra & 2.9 & $2.0-4.4$ \\
Sentir alegría al lactar & 2.6 & $1.1-6.2$ \\
Influencia de la madre & 1.3 & $1.2-1.4$ \\
\hline
\end{tabular}

\section{DISCUSIÓN}

El amamantamiento es una experiencia satisfactoria tanto para la madre como para el hijo por el contacto piel con piel que se produce entre ambos, la madre es extraordinariamente sensible durante la lactancia y su mayor preocupación gira en torno a la alimentación y cuidado de su hijo. Sin embargo, en las madres adolescentes, debido fundamentalmente a la etapa de la vida que están viviendo, al desconocimiento y falta de experiencia les provoca "angustia" (12).

El embarazo en edades cada vez más tempranas se está convirtiendo en un problema social y de salud pública de alcance mundial. Teniendo en cuenta que en el presente estudio el promedio de edad fue de 17.9 años, y en donde predomina el estado civil de unión libre, es importante tener en cuenta que la madre adolescente, es una mujer joven, necesita vivir esa etapa de su vida, ya que la maternidad temprana no la hace mujer adulta; no está preparada, en un corto tiempo debe lograr importantes metas, asumir su sexualidad, su salud reproductiva, su autocuidado, sus relaciones afectivas con la familia, con su pareja, y con su hijo. Son tareas y desafíos que las jóvenes nunca han abordado y llevan a estas madres adolescentes a sentirse recargadas de trabajo y con una carga emocional muy fuerte, lo que constituye un gran riesgo para la Lactancia Materna Exclusiva (LME).

El nivel de escolaridad materno evidenció que la gran mayoría de las adolescentes tienen educación secundaria, lo cual demuestra que a mayor nivel de instrucción materna, mayor compromiso con la LME, como los resultados encontrados en otro estudio $^{(2)}$. Lo anterior, confirma que la LME favorece el crecimiento y desarrollo del niño y cumplimiento del rol maternal y familiar.

En Colombia, la tasa de embarazos en mujeres de 15 a 19 años pasó entre 1990 y 2005 de 70 a 90 por mil y en el año 2010 disminuyó a 84 nacimientos por mil, cifra que aún continúa siendo alta, pues una de cada cinco mujeres de 15 a 19 años ha estado alguna vez embarazada, $16 \%$ ya son madres y $4 \%$ está esperando su primer hijo $^{(13)}$. El embarazo en adolescentes afecta a todos los estratos sociales, pero predomina en la clase de bajo nivel socioeconómico, en parte debido a la falta de educación sexual, y al desconocimiento de los métodos de control de la natalidad ${ }^{(14)}$. En el presente estudio se hizo evidente que la mayoría de madres adolescentes $(89.2 \%)$ pertenecientes a los estratos 1 y 2 ya habían tenido un hijo (84.2\%), dos hijos 
(14.6\%) y hasta tres hijos (1.3\%), al igual que en otro estudio en donde el $78.1 \%$ de las participantes habían tenido hijos ${ }^{(12)}$.

En cuanto a la vía del parto, en este estudio predomina el vaginal, similar a otro estudio en donde el $43 \%$ de las madres adolescentes tuvieron sus hijos por vía cesárea ${ }^{(2)}$. Sobresale en el presente estudio el cumplimiento del control prenatal en donde una gran mayoría de adolescentes tuvieron entre 5 y 8 controles, lo cual favorece la lactancia materna con el apoyo del personal de salud, como lo confirman numerosos estudios en cuanto que la información recibida por la madre durante el control prenatal y en la internación posparto, sirvió para extender la lactancia exclusiva hasta el sexto mes ${ }^{(2,12,17)}$.

Si se tiene en cuenta que la leche materna constituye el mejor alimento que puede darse en forma exclusiva a un niño o niña hasta los seis meses de edad, en un estudio realizado en Argentina ${ }^{(1)}$ la LME llegó al 6으 mes en 25\% de menores de 20 años, en otro realizado en Colombia ${ }^{(12)} 26.7 \%$ de las mujeres con hijos, dio lactancia materna durante menos de seis meses mientras que en el presente estudio se nota un incremento en la práctica de la LME en donde el 39.4\% lactó 6 meses y el $9.8 \%$ lactó más de 6 meses, contrario al estudio realizado en Canarias ${ }^{(13)}$ en donde la edad (ser muy joven) influye de forma inversa en la continuidad de la lactancia materna puesto que se observa que con respecto a las mujeres que continúan con lactancia materna a los 15 días y posteriormente, las menores de 20 años dieron menos lactancia materna al igual que en estudios realizados en Cuba ${ }^{(15,16)}$.

Como aspecto importante a considerar está el haber incluido alimentos complementarios antes de los 6 meses de vida que para este estudio el $55.4 \%$ incluyeron agua o alimentos, en tanto que en otro estudio el $28,0 \%$ de las mujeres la inició antes de los seis meses, el $49,3 \%$ a los seis meses y el $22,9 \%$ después de esta edad ${ }^{(12)}$.

Lo anterior, puede atribuirse a que el personal de salud fomenta el inicio de la LM, lo que concuerda con otras investigaciones $(1,12,17)$ en donde los momentos más utilizados por el equipo de salud y de mayor impacto para brindar información a las madres sobre alimentación natural fueron el control prenatal y la hospitalización para el parto, lo cual presupone que la mejor receptividad se da en estos escenarios que deben ser priorizados, debiéndose rescatar otros espacios como el hogar y las instancias, de control del niño sano para potenciar resultados. Además, estudios han mostrado ${ }^{(12,18)}$ que con respecto al apoyo de la lactancia materna el tiempo aumenta en las mujeres que recibieron educación sanitaria y apoyo por parte del personal de salud. Lo anterior, concuerda con los resultados del presente estudio en donde el 75.3\% recibieron información sobre Lactancia materna por parte del personal (médico y enfermera) de las instituciones de salud y un $63.3 \%$ por parte de la familia.

Es importante tener en cuenta que con respecto a las personas que recomiendan la lactancia materna exclusiva durante los primeros 6 meses de vida del niño, las instituciones, el personal de salud y la familia ${ }^{(18)}$ se convierten en los pilares fundamentales como lo muestran los resultados del presente estudio.

Tiene un interés especial el comprobar que el haber recibido información sobre lactancia materna directamente de los profesionales de salud durante la gestación aparece de forma constante en el tiempo como un factor con entidad propia, independiente del resto de factores. Lo anterior, demuestra la influencia positiva de la 
promoción de la lactancia materna desde el ámbito sanitario ${ }^{(17,19)}$ y confirma la importancia de la lactancia materna como factor protector para enfermedades prevalentes en la infancia ${ }^{(20)}$.

Desde el punto de vista psicológico, la experiencia de amamantar le permite a la madre compartir con el hijo momentos de placer y bienestar, haciendo que sea más intenso el vínculo afectivo con su bebé. La lactancia brinda un espacio de contacto íntimo, en donde el contacto corporal, el olor, la voz, las miradas, serán las primeras demostraciones de amor y de comunicación entre la madre y el bebé. Amamantar ofrece un espacio para el contacto íntimo y es una forma de compartir amor con el bebé. Es una relación sentimental basada en contacto, olor, sabor, miradas y el inicio de un diálogo que luego se producirá a través de las palabras. Es así como en el presente estudio, durante el proceso de lactancia la mayoría de madres acaricia al bebé, le habla, lo mira, mientras que unas pocas madres dicen realizar otras actividades como ver televisión entre otras. Sentimientos parecidos se encontraron en otros estudios en donde las madres consideran que lactar es un beneficio para ellas y mejora el apego de sus hijos ${ }^{(12,21)}$.

Si se tiene en cuenta que muchos estudios se han dado a la tarea de investigar sobre las causas del abandono de la lactancia materna, cabe resaltar que en uno de ellos existe una prevalencia del $93.5 \%$ del diagnóstico de enfermería lactancia materna ineficaz ${ }^{(22)}$, en donde la principal causa de suspensión de la lactancia materna al hijo anterior fue la escasa leche, presentándose en un $20.9 \%$ al igual que en otro estudio realizado ${ }^{(23)}$ donde se encontró que la causa del abandono más frecuente fue la disminución de la producción leche ${ }^{(24)}$, y por tanto, la preocupación de la madre por la producción insuficiente de leche es un factor que incide en la corta duración de la lactancia materna. Sin embargo, en el presente estudio se encontró una prevalencia menor en el abandono por causas como poca producción de leche y porque el niño se quedaba con hambre, aunque también se hallaron otras causas como preocupaciones, problemas clínicos e influencia de otras personas.

Se ha encontrado que la preocupación de la madre por la producción insuficiente de leche es un factor que incide en la corta duración de la LME ${ }^{(12)}$. Otras causas reportadas en ese estudio fueron: la enfermedad de la madre $15 \%$, el trabajo $9 \%$ y la enfermedad del niño $2 \%$. El trabajo fue la causa de suspensión de la lactancia materna en otros estudios nacionales e internacionales, sin embargo, el factor laboral que incide en la práctica de LME es la ausencia de facilidades para lactar ${ }^{(12)}$.

Diferentes estudios muestran que las madres que no recibieron apoyo familiar abandonaron con más frecuencia la lactancia materna ${ }^{(16)}$ y que dentro de otros factores encontrados estaban: la falta de apoyo de una persona cercana, la escasa producción de leche, la preocupación por el llanto del bebé porque la madre no está presente para amamantarle, el cansancio y la falta de tiempo para amamantar tranquila y sin apuro, la publicidad comercial de fórmulas lácteas que pueden reemplazar la lactancia, la falta de información y conocimientos seguros sobre la importancia de la lactancia materna, y el temor de dejar al hijo en una sala-cuna o un lugar desconocido ${ }^{(25)}$.

La situación actual de la madre trabajadora y la influencia del entorno laboral, el apoyo familiar, el empleador y los profesionales de la salud se constituyen en fuentes fundamentales para alcanzar el éxito de la lactancia materna. En el presente estudio cabe resaltar que aunque la responsabilidad como cabeza de familia recae en el 
esposo/compañero (46.8\%), un número importante de madres adolescentes son quienes responden laboralmente $(29.1 \%)$ y muchas de ellas como cabeza de familia $(12.8 \%)$, situación que se constituye en fracaso para la lactancia materna exclusiva. Sin embargo, debe tenerse en cuenta que las madres adolescentes que se encuentran estudiando (13.9\%) son dependientes de otros adultos como padres, familiares o no familiares. Además, la gran mayoría de madres adolescentes se desempeñan en el hogar por lo cual no tienen una remuneración, pero favorece en ese sentido, el contar con el tiempo suficiente para lactar porque están en el hogar. En otro estudio, cabe resaltar que el trabajo en casa se encontró como factor protector para lactar (I.C. 0.11, 0.5), lo cual expresa que las condiciones favorables del ambiente doméstico influyen para la lactancia ${ }^{(24)}$. Mientras que en diferentes estudios nacionales e internacionales el trabajo fue la causa de suspensión de la lactancia materna, sin embargo el factor laboral que incide en la práctica de LM es la ausencia de facilidades para lactar ${ }^{(12)}$.

Lo anterior, es mencionado por un estudio ${ }^{(25)}$ en cuanto a la actividad, en donde la mitad de las mujeres que fueron madres antes de los 20 años no tenían trabajo remunerado, al contrario de lo que ocurre con los padres. Para la madre trabajadora que se encuentra en periodo de amamantamiento es indispensable contar con un ambiente laboral saludable, que promueva la continuidad de la lactancia materna, facilite la relación madre- hijo-familia, mejore la calidad de vida de la familia y la comunidad, y asegure un mejoramiento en la productividad en su trabajo. Se podría decir entonces que para lograr un buen amamantamiento se requiere de tiempo, espacio, apoyo y facilidades. De igual manera, la madre adolescente, independiente de la edad, no debe estar en situación de tener que escoger entre sus diferentes roles: el ser madre lactante, madre adolescente o mujer trabajadora.

En Colombia, amamantar es un derecho, en el caso de la mujer trabajadora, el periodo de lactancia corresponde al tiempo posterior a la fecha del parto en que la mujer goza de especial protección; además, tendrá derecho al pago de las catorce (14) semanas de descanso remunerado a que hace referencia la presente ley, si no ha disfrutado de su licencia por maternidad; el artículo 238 del código sustantivo del trabajo contempla un descanso remunerado para la trabajadora durante el periodo de lactancia, el cual consiste en otorgar dos descansos diarios de 30 minutos cada uno con el fin de que la madre pueda alimentar a su bebé. Este periodo de lactancia se extiende por 6 meses posteriores a la fecha del parto, o por más meses si se cuenta con una autorización médica. Igualmente, si se cuenta con una autorización médica, el empleador debe otorgar más de dos descansos diarios a la trabajadora lactante ${ }^{(26)}$.

\section{CONCLUSIONES}

Los resultados de este estudio permitieron determinar los aspectos críticos a los cuales se ve enfrentada la madre adolescente en cuanto a los factores que dificultan la lactancia materna exclusiva y los que favorecen el abandono de la misma.

Se precisa del asesoramiento y apoyo familiar y de las instituciones de salud para que el amamantamiento sea una experiencia satisfactoria tanto para la madre adolescente como para el hijo favoreciendo no solo el contacto piel con piel que se produce entre ambos, sino porque la madre es extraordinariamente sensible durante la lactancia y prioriza sus demandas sobre las necesidades y cuidados de su hijo. 
Para la madre que estudia y/o trabaja que se encuentra en periodo de amamantamiento es indispensable contar con un ambiente saludable, que promueva la continuidad de la lactancia materna exclusiva, facilite la relación madre- hijo-familia, mejore la calidad de vida de la familia y la comunidad.

\section{REFERENCIAS BIBLIOGRÁFICAS}

1. Armelini P. El $22 \%$ de los bebes dejan de ser amamantados al mes de vida. Disponible en: URL: : http://med.unne.edu.ar/revista/revista177/1 177.pdf

2. González IA, Huespe MS, Auchter MC. Lactancia materna exclusiva factores de éxito y/o fracaso. Revista de Posgrado de la Vla Cátedra de Medicina, Enero 2008; 177: 2

3. Colombia, Ministerio de Salud y Protección Social. Lactancia materna articulada a la estrategia "De Cero a Siempre". Boletín de prensa N²09 de 2012. Disponible en: URL:

http://www.minsalud.gov.co/Paginas/Lactancia\%20materna\%20articulada\%20a\%20la \%20estrategia\%20de\%20\%E2\%80\%9CCero\%20a\%20siempre\%E2\%80\%9D\%20pide \%20Minsalud.aspx

4. Ministerio de Salud y Protección Social. Atención integral a la primera infancia. Disponible en:

URL:

http://www.deceroasiempre.gov.co/QuienesSomos/Documents/DE-CERO-A-

SIEMPRE-Version-Comunidad-Academica-2011.pdf

5. Encuesta nacional de Demografía y Salud- ENDS 2010. Disponible en: URL: http://www.profamilia.org.co/encuestas/Profamilia/Profamilia/index.php?option=com c ontent\&view=article\&id=97\&ltemid=109

6. Castillo V, Lamas R. Lactancia materna un desafío para las madres adolescentes. Módulo I: Tendencias en Salud Pública: Salud Familiar y Comunitaria y Promoción. La Serena, marzo - abril del 2005 1-14 Disponible en: URL: http://medicina.uach.cl/saludpublica/diplomado/contenido/trabajos/1/La\%20Serena\%2 02005/Lactancia_materna_un_desafio_en_madres_adolescentes.pdf

7 Durán $R$, et al. Factores psicosociales que influyen en el abandono de la lactancia materna. Rev Cubana Pediatr 1999; 71 (2):73-9

8. CDC. Epi Info, v. 6.04d - StatCalc. Epidemiología en ordenadores. Atlanta, Georgia. Enero, 2001

9. StataCorp. Stata Statistical Software: Release 10.1. College Station, Texas: Stata Corporation. 2008

10. Alegre Palomino Y, Suárez Bustamante M. Instrumentos de Atención a la Familia: EI Familiograma y el APGAR familiar. RAMPA 2006. 1(1):48 - 57

11. República de Colombia, Ministerio de Salud, Resolución N 8430 de 1993 (4 de octubre de 1993). Por la cual se establecen las normas científicas, técnicas y administrativas para la investigación en salud. Disponible en: URL: http://comitedeetica.clinicalili.org/docs/RESL8430.pdf

12. Gamboa ED, López N, Prada GE, Gallo KY. Conocimientos, actitudes y prácticas relacionados con lactancia maternal en mujeres en edad fértil en una población vulnerable. Rev Chil Nutr 2008; 35 (1): 43-52

13. República de Colombia, Departamento Nacional de Planeación. Consejo Nacional de Política Económica y Social, Lineamientos para el desarrollo de una estrategia para la prevención del embarazo en la adolescencia y la promoción de proyectos de vida para los niños, niñas, adolescentes y jóvenes en edades entre 6 y 19 años. Documento Conpes Social 147. Bogotá, D.C., Enero 31 de 2912. Disponible en URL: http://www.dnp.gov.co/LinkClick.aspx?fileticket=BSINpiuEDQ0\%3D\&tabid=1473 
14. López Rodríguez Y. Embarazo en la adolescencia y su repercusión biopsicosocial sobre el organismo de la madre y de su futuro hijo. Revista Cubana de Enfermería. 2012; 28(1):23-36

15. Herrero LA, Gorrita RR ¿Cómo influyen diferentes factores psicosociales en el abandono precoz de la lactancia materna? Revista de Ciencias Médicas La Habana 2009; 15 (3): 28-34

16. Durán R, Villegas D, Sobrado Z, Almanza M. Factores psicosociales que influyen en el abandono de la lactancia materna. Rev Cubana Pediatr 1999; 71(2):72-9

17. Estévez MD, Martell D. Medina R, García E, Saavedra P. Factores relacionados con el abandono de la lactancia materna. Universidad de Las Palmas de Gran Canaria. Hospital Universitario Materno-Infantil de Canarias. An Esp Pediatr 2002; 56: 144-150

18. García Casanova MC, García Casanova S, Pi Juan M, Ruiz Mariscal E y Parellada Esquius N. Lactancia materna: ¿puede el personal sanitario influir positivamente en su duración? Aten Primaria 2005; 35 (6):295-300.

19. Wambach KA, Aaronson L, Breedlove G, Domian EW, Rojjanasrirat W , Yeh HW.

Un ensayo controlado aleatorio de apoyo a la lactancia y educación para madres adolescentes. West J Nurs Res 2011; 33 (4): 486-505

20. Alzate Meza MC, Arango C, Castaño JJ et al. Lactancia materna como factor protector para enfermedades prevalentes en niños hasta de 5 años de edad en algunas instituciones educativas de Colombia 2009. Estudio trasversal. Rev. Colombiana de obstetricia y ginecología 2011; 62 (1): 57-63

21. Muñoz LP, Martínez JW, Quintero AR, Lasso Gl, Medina GA. Características asociadas a las prácticas de lactancia en madres asistentes a tres instituciones de Salud de Pereira *2004. Investigaciones Andina 2005, 11: 17-27

22. Pinilla E, Orozco LC, Camargo FA, Alfonso EP, Peña ER, Villabona LN. Acevedo Y. Lactancia materna ineficaz: prevalencia y factores asociados. Salud UIS 2011; 43 (3): 271-279

23. Chávez Merlos A, Sánchez GR, Ortiz OH, Peña Ortega B, Arocha Reyes B. Causas de abandono de la lactancia materna. Rev. Fac. Med. UNAM 2002; 45 (2): 5355.

24. Muñoz LP, Martínez JW, Quintero AR, Lasso Gl, Medina GA. Características asociadas a las prácticas de lactancia en madres asistentes a tres instituciones de salud de Pereira. Investigaciones Andina 2005; 11: 17-27

25. Cerda Muñoz L. La madre que amamanta y el entorno laboral. Matronas Prof. 2008; 9 (1): 21-26

26. Colombia. Código sustantivo del trabajo. Disponible en URL: http://www.secretariasenado.gov.co/senado/basedoc/codigo/codigo sustantivo trabai o pr008.html

ISSN 1695-6141

(C) COPYRIGHT Servicio de Publicaciones - Universidad de Murcia 